\title{
Read-through transcripts in normal human lung parenchyma are down-regulated in lung adenocarcinoma
}

\author{
Giulia Pintarelli ${ }^{1, *}$, Alice Dassano ${ }^{1, *}$, Chiara E. Cotroneo ${ }^{1,6}$, Antonella Galvan ${ }^{7}$ \\ Sara Noci ${ }^{1}$, Rocco Piazza ${ }^{2,3}$, Alessandra Pirola ${ }^{2}$, Roberta Spinelli ${ }^{8}$, Matteo Incarbone ${ }^{4}$, \\ Alessandro Palleschi ${ }^{5}$, Lorenzo Rosso ${ }^{5}$, Luigi Santambrogio ${ }^{5}$, Tommaso A. Dragani ${ }^{1}$, \\ Francesca Colombo ${ }^{1}$ \\ ${ }^{1}$ Department of Predictive and Prevention Medicine, Fondazione IRCCS, Istituto Nazionale dei Tumori, Milan, Italy \\ ${ }^{2}$ Department of Health Sciences, University of Milano-Bicocca, Monza, Italy \\ ${ }^{3}$ Hematology and Clinical Research Unit, San Gerardo Hospital, Monza, Italy \\ ${ }^{4}$ Department of Surgery, San Giuseppe Hospital, Multimedica, Milan, Italy \\ ${ }^{5}$ Department of Surgery, IRCCS Fondazione Cà Granda Ospedale Maggiore Policlinico, Università degli Studi di Milano, Milan, \\ Italy \\ ${ }^{6}$ Present Address: UCD School of Biomolecular and Biomedical Science, University College Dublin, Belfield, Dublin, Ireland \\ ${ }^{7}$ Formerly, Department of Predictive and Prevention Medicine, Fondazione IRCCS, Istituto Nazionale dei Tumori, Milan, Italy \\ ${ }^{8}$ Formerly, Department of Health Sciences, University of Milano-Bicocca, Monza, Italy \\ *These authors have contributed equally to this work \\ Correspondence to: Tommaso A. Dragani, e-mail: tommaso.dragani@istitutotumori.mi.it
}

Keywords: conjoined genes, gene fusion, lung adenocarcinoma, RNA-Seq, read-through transcripts

Received: November 17, 2015

Accepted: February 18, 2016

Published: April 02, 2016

\section{ABSTRACT}

Read-through transcripts result from the continuous transcription of adjacent, similarly oriented genes, with the splicing out of the intergenic region. They have been found in several neoplastic and normal tissues, but their pathophysiological significance is unclear. We used high-throughput sequencing of cDNA fragments (RNA-Seq) to identify read-through transcripts in the non-involved lung tissue of 64 surgically treated lung adenocarcinoma patients. A total of 52 distinct read-through species was identified, with 24 patients having at least one read-through event, up to a maximum of 17 such transcripts in one patient. Sanger sequencing validated 28 of these transcripts and identified an additional 15, for a total of 43 distinct read-through events involving 35 gene pairs. Expression levels of 10 validated read-through transcripts were measured by quantitative PCR in pairs of matched non-involved lung tissue and lung adenocarcinoma tissue from 45 patients. Higher expression levels were observed in normal lung tissue than in the tumor counterpart, with median relative quantification ratios between normal and tumor varying from 1.90 to 7.78; the difference was statistically significant $(P<0.001$, Wilcoxon's signed-rank test for paired samples) for eight transcripts: ELAVL1-TIMM44, FAM162B-ZUFSP, IFNAR2-IL10RB, INMT-FAM188B, KIAA1841-C2orf74, NFATC3-PLA2G15, SIRPB1-SIRPD, and SHANK3-ACR. This report documents the presence of read-through transcripts in apparently normal lung tissue, with inter-individual differences in patterns and abundance. It also shows their down-regulation in tumors, suggesting that these chimeric transcripts may function as tumor suppressors in lung tissue. 


\section{INTRODUCTION}

Several genetic alterations have been reported to act as driver events in lung tumorigenesis or to modulate the progression of lung tumors and their responses to therapy [1,2]. Among these alterations, chromosomal rearrangements (e.g. translocations, inversions and insertions) affecting genes encoding receptor tyrosine kinases, such as $A L K, R O S I$ and $R E T$, have been extensively studied [1, 3-9]. These rearrangements can give rise to gene fusions by juxtapositioning previously independent coding sequences; when these fused genes are transcribed, they produce chimeric transcripts.

Chimeric transcripts can also be generated in the absence of chromosomal rearrangements, as a result of the transcriptional "read through" of two adjacent, similarly oriented genes, with the splicing out of the intergenic region [10]. The identification of read-through transcripts has been made easier by the possibility of transcriptome sequencing using next-generation technologies [11]. Through this approach, read-through transcripts have been observed in several tumor types, such as breast, prostate, gastric, and renal cancer [12-18]. Read-through transcripts in cancer tissues may have tumorigenic potential, since their silencing in cancer cell lines reduces cell proliferation [13-15]. In lung cancer, evidence for read-through transcripts is currently limited to our incidental discovery, during a gene expression study, of RNA chimeras formed by the intergenic transcription of the conjoined genes PPP $3 R 1$ and $C N R I P 1$, in a few samples of lung adenocarcinoma and normal lung tissue [19].

Even though the presence of read-through transcripts in normal, non-neoplastic tissue is documented $[10,12,18,20-23]$, their physiological role is still unknown. Computational and experimental analyses suggested that chimeric transcripts, including read-throughs, are translated, producing fusion proteins with altered properties, such as new intracellular localizations and new functions, through the novel combination of protein domains [20,24]. The biological functions of fusion proteins encoded by some read-through transcripts have been primarily investigated in cancer cell lines, in order to understand their role in tumorigenesis: indeed, the effects of read-through transcript silencing on cell proliferation, colony formation, and ability to grow in an anchorage-independent manner (soft agar assay) have been reported [13, 14, 17, 25]. It has also been suggested that untranslated read-through transcripts regulate gene expression at the RNA level, as either non-coding RNA or regulatory RNA [22].

In this study, we used RNA sequencing to search for additional read-through transcripts in normal lung parenchyma from adenocarcinoma patients. Candidate transcripts were examined by PCR followed by Sanger sequencing of the read-through fusion points, permitting the validation of 43 transcripts. Comparison of the expression levels of 10 of these read-through transcripts in paired samples of non-involved lung tissue and lung adenocarcinoma tissue revealed lower levels in tumor tissue than in the normal tissue counterpart.

\section{RESULTS}

\section{Detection of read-through fusion events}

Paired-end whole transcriptome sequencing (RNA-Seq) was carried out on 64 samples of RNA from non-involved lung tissue of 64 lung adenocarcinoma patients (Table 1). The RNA-Seq data were analyzed to identify chimeric transcripts involving two adjacent genes on the same chromosome, in the same orientation. This criterion, together with the fact that the RNA samples were from apparently normal tissue, maximized the probability that the identified chimeric transcripts had been generated by transcriptional read-through events, without genetic aberrations. Among the 64 samples analyzed, 24 had at least one read-through transcript, up to a maximum of 17 different read-through transcripts observed in a single patient. Altogether we found 52 unique read-through species involving 37 different pairs of adjacent genes (Table 2). In some cases, the read-through events involved different exons of the same pair of genes. Most read-through transcripts were found in only one or two patients, but eight were found in 3-10 patients.

\section{Validation of the detected read-through fusion events}

To confirm the existence of the read-through transcripts identified by RNA-Seq, we carried out PCR, using primers to amplify the sequence spanning the junction, on cDNA from at least one patient in whom each transcript was identified. For four transcripts, PCR did not amplify any sequence so these were excluded from further analysis. For 17 transcripts, we obtained multiple amplicons of different sizes, while for the remaining 31 transcripts we obtained a single amplicon, with 21 of them having the expected molecular weight. Sanger sequencing of the amplicons validated 28 transcripts as predicted by RNA-Seq and excluded seven of them (Table 2). Moreover, for the remaining 13 transcripts, Sanger sequencing did not confirm the predicted fusion point, but instead identified one or two different splicing isoforms involving different exons of the same conjoined genes (Table 3 ). Hence this analysis validated 28 read-through transcripts among the 52 discovered using RNA-Seq and identified 15 additional read-through transcripts by Sanger sequencing of PCR products, for a total of 43 read-through events involving 35 pairs of genes.

Read-through transcription most frequently involved the second-to-last exon of the first gene, i.e. the one before the $3^{\prime}$-UTR (in 28 of 43 cases, $\sim 65 \%$ ), and the second exon of the second gene (in 30 cases, $\sim 70 \%$ ). 
Table 1: Clinical characteristics of the 64 lung adenocarcinoma patients from whom total RNA from resected non-tumor lung tissue was analyzed by RNA-Seq and of 45 lung adenocarcinoma patients from whom total RNA from non-tumor and tumor lung tissue was analyzed by qPCR (these 45 patients included 6 patients analyzed by RNA-Seq)

\begin{tabular}{|l|c|c|}
\hline \multicolumn{2}{|c|}{ RNA-Seq } & qPCR \\
\hline Sex, $n(\%)$ & & $34(75.6)$ \\
\hline Men & $50(78.1)$ & $11(24.4)$ \\
\hline Women & $14(21.9)$ & $62.6(10.7)$ \\
\hline Age at surgery, years, mean (SD) & $63.8(7.3)$ & 31 \\
\hline Smoking status, $n$ & & 11 \\
\hline Smoker & 61 & 3 \\
\hline Non-smoker & 1 & \\
\hline Missing & 2 & 18 \\
\hline Stage, $n$ & & 8 \\
\hline I & 55 & 13 \\
\hline II & 3 & 6 \\
\hline III or IV & 5 & \\
\hline Missing & 1 & \\
\hline
\end{tabular}

Sometimes, however, the read-through event was more complex. For instance, in the CD59-C11orf91 transcript, a portion of an intragenic (intronic) region was included. Moreover, in two cases, incomplete exons were found: HARS2 exon 13 in HARS2-ZMAT2 and SEC31B exon 2 in NDUFB $-S E C 31 B$. The median intergenic distance, calculated as the number of base pairs between the end of the first parent gene locus and the start of the second parent gene locus, was 3,941 bp. For 10 gene pairs the intergenic distance was greater than $10 \mathrm{~kb}$, whereas in seven cases it was less than $1 \mathrm{~kb}$. Four validated read-though transcripts originated from overlapping genes. Finally, 17 read-through events maintained the original open reading frames of the two coding sequences that were joined (Tables 2 and 3 ).

\section{Expression levels of read-through transcripts in matched samples of non-involved and lung adenocarcinoma tissue}

The expression levels of the 43 validated read-through transcripts in non-involved lung tissue were generally low (not shown). To examine their expression levels in lung adenocarcinoma tissue, we focused on 10 transcripts whose levels were among the more abundant and for which only a single amplicon was obtained at PCR in the validation step. These transcripts were: $C H I A-P I F O$, CTSC-RAB38, ELAVL1-TIMM44, FAM162B-ZUFSP, IFNAR2(exon 7)-IL10RB(exon 2), INMT-FAM188B, KIAA1841-C2orf74, NFATC3-PLA2G15, SHANK3-ACR, and SIRPB 1-SIRPD. We measured, by quantitative PCR, the expression levels of these read-through transcripts in up to 45 pairs of matched tissues of non-tumor lung parenchyma and lung adenocarcinoma tissue. Not all transcripts were measurable in all pairs of samples, and we excluded from further analyses those pairs for which qPCR data were unreliable (i.e. cycle threshold standard deviation $>0.5$ ) in at least one sample. In all cases the median fusion transcript level was higher in normal lung tissue than in the tumor counterpart, and the difference was significant in eight cases (Figure 1). The median relative quantification ratios between normal and tumor varied from 1.90 (IFNAR2-IL10RB) to 7.78 (SHANK3-ACR). In detail, the difference of expression was statistically significant for ELAVL1-TIMM44 $\left(P=1.0 \times 10^{-5}, n=25\right)$, FAM162B-ZUFSP $\left(P=3.8 \times 10^{-6}, n=19\right)$, IFNAR2-IL10RB $\left(P=1.5 \times 10^{-5}, n=25\right)$, INMT-FAM188B $\left(P=5.7 \times 10^{-7}\right.$, $n=26)$, KIAA1841-C2orf74 $\left(P=3.0 \times 10^{-5}, n=24\right)$, NFATC3-PLA2G15 $\left(P=1.9 \times 10^{-4}, n=28\right)$, SIRPB1-SIRPD $\left(P=4.8 \times 10^{-7}, n=23\right)$, and SHANK3-ACR $\left(P=1.9 \times 10^{-7}\right.$, $n=33$; Wilcoxon's signed-rank test for paired samples; Figure 1)

To determine if the expression levels of these 10 read-through transcripts were affected by the patients' clinical characteristics, we fitted a multivariate linear model, treating sex, smoking habit, and disease stage (stage I or stage > I) as covariates (Table 4). This analysis confirmed that the expression levels were significantly associated with the type of tissue (normal vs. tumor) for the eight fusion transcripts and also found a significant effect for a ninth, namely CHIA-PIFO $(P=0.0184)$. Read-through transcript levels were not affected by smoking habit. FAM162B-ZUFSP read-through levels were significantly associated with disease stage and sex ( $P=0.0015$ and $P=0.019$, respectively), whereas those of NFATC3-PLA2G15 were associated with sex $(P=0.040)$. The expression levels of the other read-through transcripts were not affected by sex or stage. 
Table 2: Read-through transcripts identified by RNA-Seq analysis

\begin{tabular}{|c|c|c|c|c|c|c|c|}
\hline $\begin{array}{l}\text { Read-through } \\
\text { name }^{1}\end{array}$ & Chr. $^{2}$ & $\begin{array}{l}\text { First transcript } \\
(\text { exon })^{3}\end{array}$ & $\begin{array}{l}\text { Second transcript } \\
(\text { exon })^{3}\end{array}$ & $\begin{array}{c}\text { Intergenic } \\
\text { region (bp) }\end{array}$ & $\begin{array}{c}\text { N. } \\
\text { patients }^{4}\end{array}$ & $\begin{array}{c}\text { PCR } \\
\text { validated }\end{array}$ & $\begin{array}{c}\text { Maintained } \\
\text { frame }\end{array}$ \\
\hline $\begin{array}{l}\text { AGRP- } \\
\text { ATP6V0D1 }\end{array}$ & 16 & NM_001138(3) & NM_004691 (2) & 1,334 & 1 & Yes & No \\
\hline ARF3-FKBP11 & 12 & NM_001659(4) & NM_001143781 (4) & 9,249 & 1 & Different $^{6}$ & See Table 3 \\
\hline ARPC4-TTLL3 & 3 & NM_001024959 (5) & NM_001025930(2) & 360 & 10 & Yes & No \\
\hline CD59-C11orf91 & 11 & NM_203331 (4) & NM_001166692 (2) & $\mathrm{NA}^{5}$ & 2 & Different $^{6}$ & See Table 3 \\
\hline CHIA-PIFO & 1 & NM_021797 (8) & NM_181643(2) & 25,722 & 1 & Yes & Yes \\
\hline \multirow[t]{2}{*}{ CTSC-RAB38 } & \multirow[t]{2}{*}{11} & NM_001814(5) & NM_022337 (2) & \multirow[t]{2}{*}{118,125} & 2 & Yes & Yes \\
\hline & & NM_001814(4) & NM_022337 (2) & & 1 & No & - \\
\hline \multirow[t]{2}{*}{ CTSD-IFITM10 } & \multirow[t]{2}{*}{11} & NM_001909 (8) & NM_001170820 (2) & \multirow[t]{2}{*}{2,161} & 3 & Yes & Yes \\
\hline & & NM_001909(7) & NM_001170820(2) & & 2 & No & - \\
\hline $\begin{array}{l}\text { ELAVL1- } \\
\text { TIMM44 }\end{array}$ & 19 & NM_001419(4) & NM_006351 (3) & 14,658 & 2 & Different $^{6}$ & See Table 3 \\
\hline $\begin{array}{l}\text { FAM162B- } \\
\text { ZUFSP }\end{array}$ & 6 & NM_001085480(3) & NM_145062(2) & 83,406 & 1 & Yes & No \\
\hline FARSA-SYCE2 & 19 & NM_004461 (12) & NM_001105578(3) & 3,203 & 1 & Yes & Yes \\
\hline HARS2-ZMAT2 & 5 & NM_012208(13) & NM_144723 (2) & $\mathrm{NA}^{5}$ & 1 & Different $^{6}$ & See Table 3 \\
\hline $\begin{array}{l}\text { HSD17B11- } \\
\text { HSD17B13 }\end{array}$ & 4 & NM_016245(5) & NM_178135(2) & 13,704 & 1 & Different $^{6}$ & See Table 3 \\
\hline \multirow[t]{2}{*}{ IFNAR2-IL10RB } & \multirow[t]{2}{*}{21} & NM_207584(7) & NM_000628(2) & \multirow[t]{2}{*}{683} & 1 & Yes & Yes \\
\hline & & NM_207584(7) & NM_000628(3) & & 1 & Yes & No \\
\hline \multirow[t]{3}{*}{ INMT-FAM188B } & \multirow[t]{3}{*}{7} & NM_001199219(2) & NM_032222(2) & \multirow[t]{3}{*}{13,815} & 4 & Yes & No \\
\hline & & NM_001199219(2) & NM_032222(3) & & 7 & No & - \\
\hline & & NM_001199219(3) & NM_032222(3) & & 1 & $\mathrm{No}^{7}$ & - \\
\hline $\begin{array}{l}\text { KIAA1841- } \\
\text { C2orf74 }\end{array}$ & 2 & NM_032506(21) & NM_001143960 (2) & $\mathrm{NA}^{5}$ & 1 & Yes & No \\
\hline LMAN2-MXD3 & 5 & NM_006816(7) & NM_001142935(4) & 18,805 & 1 & Yes & Yes \\
\hline MBD1-CFAP53 & 18 & NM_001204139(14) & NM_145020(2) & 360 & 1 & Yes & No \\
\hline MED22-SURF6 & 9 & NM_133640(4) & NM_006753 (2) & 1,925 & 1 & Yes & No \\
\hline MRPS17-GBAS & 7 & NM_015969(1) & NM_001202469(3) & $\mathrm{NA}^{5}$ & 1 & $\mathrm{No}^{7}$ & - \\
\hline NAA60-CLUAP1 & 16 & NM_001083601(1) & NM_015041(2) & 13,961 & 1 & Different $^{6}$ & See Table 3 \\
\hline $\begin{array}{l}\text { NDUFB8- } \\
\text { SEC31B }\end{array}$ & 10 & NM_005004 (4) & NM_015490(2) & $\mathrm{NA}^{5}$ & 1 & Yes & No \\
\hline $\begin{array}{l}\text { NFATC3- } \\
\text { PLA2G15 }\end{array}$ & 16 & NM_173165(9) & NM_012320(2) & 16,045 & 2 & Yes & Yes \\
\hline \multirow[t]{5}{*}{ NKX2-1-SFTA3 } & \multirow[t]{5}{*}{14} & NM_001079668(2) & NM_001101341 (4) & \multirow[t]{5}{*}{2,568} & 5 & Yes & No \\
\hline & & NM_001079668(2) & NM_001101341(2) & & 2 & Yes & No \\
\hline & & NM_001079668 (1) & NM_001101341 (2) & & 1 & Yes & No \\
\hline & & NM_001079668 (1) & NM_001101341 (4) & & 3 & Different $^{6}$ & See Table 3 \\
\hline & & NM_001079668 (1) & NM_001101341 (3) & & 5 & No & - \\
\hline \multirow{2}{*}{$\begin{array}{l}\text { PACSIN2- } \\
\text { ARFGAP3 }\end{array}$} & \multirow[t]{2}{*}{22} & NM_001184970(10) & NM_001142293 (3) & \multirow[t]{2}{*}{$\mathrm{NA}^{5}$} & 1 & Yes & No \\
\hline & & NM_001184970(10) & NM_001142293 (4) & & 1 & Different $^{6}$ & See Table 3 \\
\hline PIR-FIGF & $\mathrm{X}$ & NM_001018109 (9) & NM_004469 (2) & 423 & 1 & Yes & No \\
\hline \multirow{2}{*}{$\begin{array}{l}\text { PLEKHO2- } \\
\text { ANKDD1A }\end{array}$} & \multirow[t]{2}{*}{15} & NM_001195059 (2) & NM_182703 (5) & \multirow[t]{2}{*}{43,895} & 1 & Yes & Yes \\
\hline & & NM_001195059(4) & NM_182703 (5) & & 1 & Different $^{6}$ & See Table 3 \\
\hline PPRC1-NOLC1 & 10 & NM_015062(13) & NM_004741 (4) & 1,851 & 1 & Different $^{6}$ & See Table 3 \\
\hline
\end{tabular}




\begin{tabular}{|c|c|c|c|c|c|c|c|}
\hline \multirow{5}{*}{$\begin{array}{l}\text { SCNN1A- } \\
\text { TNFRSF1A }\end{array}$} & \multirow[t]{5}{*}{12} & NM_001159575 (12) & NM_001065(2) & \multirow[t]{5}{*}{4,729} & 2 & Yes & Yes \\
\hline & & NM_001159575 (12) & NM_001065 (3) & & 2 & No & - \\
\hline & & NM_001159575(13) & NM_001065(2) & & 1 & $\mathrm{No}^{7}$ & - \\
\hline & & NM_001159575(10) & NM_001065(2) & & 1 & No & - \\
\hline & & NM_001159575(9) & NM_001065 (2) & & 1 & No & - \\
\hline SFTPC-BMP1 & 8 & NM_003018(3) & NM_001199(2) & 257 & 2 & Yes & No \\
\hline SHANK3-ACR & 22 & NM_033517(22) & NM_001097(2) & 4,898 & 1 & Yes & Yes \\
\hline SIRPB1-SIRPD & 20 & NM_001083910(2) & NM_178460(2) & 4,678 & 1 & Yes & Yes \\
\hline SNTB2-VPS4A & 16 & NM_006750(6) & NM_013245(3) & 2,304 & 1 & Different $^{6}$ & See Table 3 \\
\hline $\begin{array}{l}\text { SPECC1L- } \\
\text { ADORA2A }\end{array}$ & 22 & NM_015330(13) & NM_000675(3) & 139 & 1 & Different $^{6}$ & See Table 3 \\
\hline TSTD1-F11R & 1 & NM_001113205(1) & NM_016946(3) & 16,283 & 2 & Different $^{6}$ & See Table 3 \\
\hline VAMP8-VAMP5 & 2 & NM_003761 (2) & NM_006634 (2) & 2,377 & 5 & Yes & Yes \\
\hline ZDHHC1-TPPP3 & 16 & NM_013304 (10) & NM_016140(3) & 884 & 1 & Yes & No \\
\hline ZNF343-SNRPB & 20 & NM_024325 (6) & NM_198216(2) & 10,964 & 1 & $\mathrm{No}^{7}$ & - \\
\hline
\end{tabular}

${ }^{1}$ Gene1-Gene2 symbol.

${ }^{2}$ Chr.: chromosome.

${ }^{3}$ Exons joined by the read-through event.

${ }^{4}$ Number of patients in which the read-through transcript was identified by bioinformatic analysis of RNA-Seq data.

${ }^{5} \mathrm{NA}$ : not available; there is no intergenic distance since genes are overlapping.

${ }^{6}$ Identification of different read-through fusion points by Sanger-sequencing (see Table 3).

${ }^{7}$ No sequence amplified at PCR.

Table 3: Read-through transcripts identified by Sanger sequencing of PCR amplicons

\begin{tabular}{|c|c|c|c|}
\hline Read-through name ${ }^{1}$ & First transcript (exon) ${ }^{2}$ & Second transcript (exon) ${ }^{2}$ & Maintained frame \\
\hline \multirow{2}{*}{ ARF3-FKBP11 } & NM_001659(4) & NM_001143781 (2) & No \\
\hline & NM_001659(4) & NM_001143781 (3) & No \\
\hline CD59-C11 orf91 & NM_203331(4)* & NM_001166692(2)* & No \\
\hline ELAVL1-TIMM44 & NM_001419(4) & NM_006351 (2) & No \\
\hline HARS2-ZMAT2 & NM_012208 (13)§ & NM_144723 (2) & No \\
\hline HSD17B11-HSD17B13 & NM_016245(6) & NM_178135(2) & No \\
\hline NAA60-CLUAP1 & NM_001083601 (2) & NM_015041 (2) & No \\
\hline NKX2-1-SFTA3 & NM_001079668(1) & NM_001101341 (2) & No \\
\hline PACSIN2-ARFGAP3 & NM_001184970(10) & NM_001142293 (2) & No \\
\hline \multirow{2}{*}{ PLEKHO2-ANKDD1A } & NM_001195059(5) & NM_182703 (4) & Yes \\
\hline & NM_001195059(5) & NM_182703 (5) & Yes \\
\hline PPRC1-NOLC1 & NM_015062(13) & NM_004741 (2) & No \\
\hline SNTB2-VPS4A & NM_006750 (6) & NM_013245 (2) & Yes \\
\hline SPECC1L-ADORA2A & NM_015330(14) & NM_000675 (3) & Yes \\
\hline TSTD1-F11R & NM_001113205 (1) & NM_016946(2) & Yes \\
\hline
\end{tabular}

${ }^{1}$ Gene1-Gene2 symbol.

${ }^{2}$ Exons joined by the read-through event.

*A portion of intragenic region is included in the read-through transcript.

§Incomplete exon. 


\section{DISCUSSION}

In this study we searched for read-through transcripts, expressed in the non-involved lung tissue of 64 lung adenocarcinoma patients, by RNA-Seq analysis. We confirmed by Sanger sequencing 43 read-through events, involving 35 pairs of conjoined genes. For 10 of these validated read-through transcripts, quantitative PCR analyses in paired samples of non-involved and tumor tissue revealed that nine were down-regulated in lung adenocarcinoma tissue.

Considering the 35 gene pairs involved in read-through events, 26 had already been reported in the literature $[10,12,14,21,22,26]$ or annotated in one or more of the NCBI (http://www.ncbi.nlm.nih.gov/ gene/), Ensembl (http://grch37.ensembl.org/index.html), ConjoinG (http://metasystems.riken.jp/conjoing/index), and UCSC (https://genome-euro.ucsc.edu/cgi-bin/hg Gateway? redirect $=$ manual\&source $=$ genome.ucsc.edu) databases (Supplementary Table S1). In particular, for 16 gene pairs, the exact read-through fusion point identified in this study had already been described, and for six of them (INMT-FAM188B, MED22-SURF6, NDUFB8-SEC31B, SCNN1A-TNFRSF1A, SIRPB1-SIRPD, and SNTB2$V P S 4)$ the transcripts had already been detected in lung, as indicated by ConjoinG database. For an additional 10 gene pairs, the reported read-through events involved different exons and generated different read-through transcript isoforms. Because of the stringent selection criteria used to select chimeric transcripts (using FusionAnalyser software for RNA-Seq data mining) in our study, it is possible that additional read-through transcripts are present in normal lung tissue and deserve further investigation; indeed, by
PCR amplification, besides the candidate read-through transcripts undergoing validation, we detected additional read-through isoforms.

Features of the read-through events identified in this study are comparable with those reported for other read-through transcripts in other tissues. In particular, the median intergenic distance of our read-through fusion events $(3,941 \mathrm{bp})$ is small in comparison to that for all gene pairs in the human genome (estimated as $48 \mathrm{~kb}$ [10] and $64 \mathrm{~kb}$ [12]); this pattern was already observed in earlier studies on conjoined genes, which reported intergenic distances of $8.5-10 \mathrm{~kb}[10,12,22]$. This recurrent finding indicates that read-through events occur more frequently between close genes. Also, the frequent involvement of the exon just before the 3'-UTR of the first parent gene and of the second exon of the second gene in the read-through fusion events has already been observed in other studies of read-through events in different tissues $[10,22]$.

Almost half of the identified read-through fusion events $(\sim 42 \%)$ respected the original open reading frame of the two coding sequences that were joined. This percentage is slightly higher than the $25 \%$ observed by Akiva et al. [10]. When the reading frame is maintained, it is possible that a bifunctional fusion protein, containing domains of both the original proteins, can be produced, as already demonstrated for TWE-PRIL and Kua-UEV1 proteins $[27,28]$. In other cases, when the read-through event causes a frame shift, new proteins may be created with an intact part of the first parent protein fused to an alternative isoform of the second. Even when read-through transcripts are not translated into protein, they may have a role in regulating the expression of the parent genes [10].
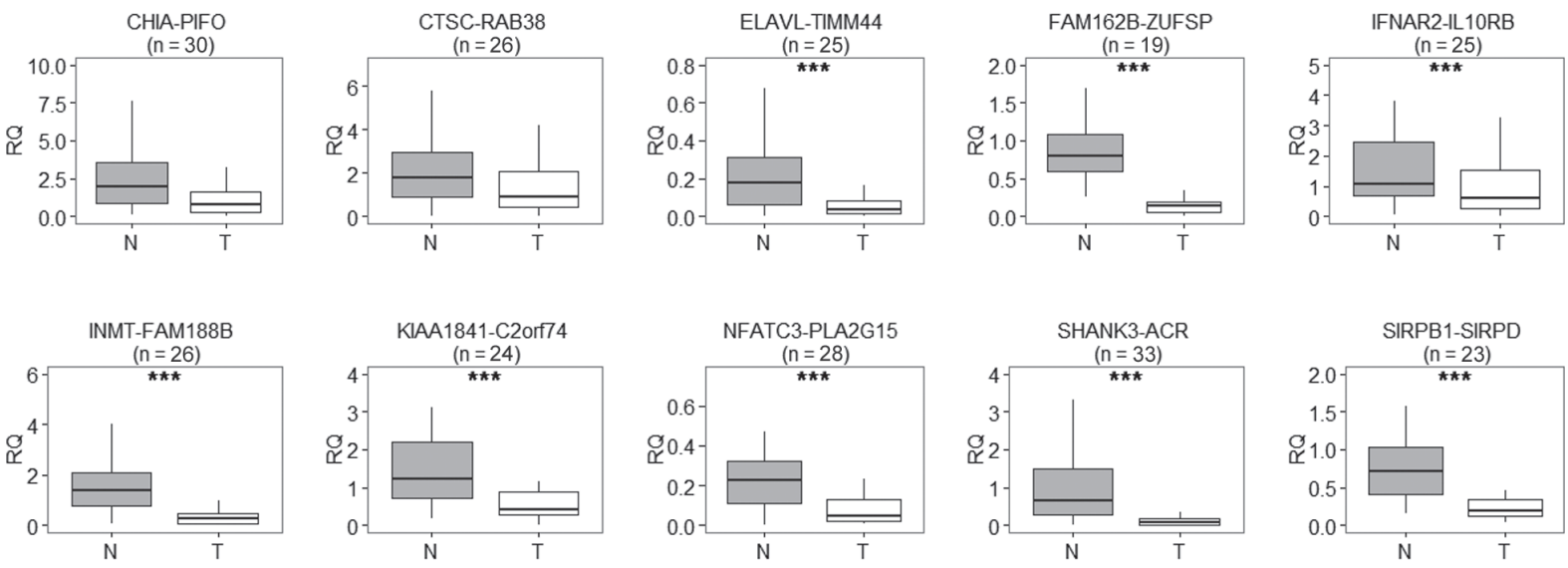

Figure 1: Relative quantification (RQ) of read-through transcripts, in pairs of matched non-involved and tumor tissue from lung adenocarcinoma patients, reveals that read-through transcripts were expressed at lower levels in tumor tissue $(T)$ than in the normal counterpart $(N) ; \boldsymbol{n}=$ number of normal-tumor tissue pairs examined. The line within each box represents the median RQ; upper and lower edges of each box are 75th and 25th percentiles, respectively; top and bottom whiskers indicate the greatest and least RQ values, respectively. ${ }^{* * *} P<0.001$, paired Wilcoxon's signed-rank test for paired samples. 
Table 4: Influence of clinical characteristics on read-through transcript levels in lung adenocarcinoma tissue versus non-involved lung tissue, determined by testing in a multivariate linear model, using disease stage, smoking habit and sex as covariates

\begin{tabular}{|c|c|c|c|c|}
\hline Read-through ${ }^{1}$ & Variable/Covariate ${ }^{2}$ & Coefficient estimate & $\mathbf{S E}^{3}$ & $P^{4}$ \\
\hline \multicolumn{5}{|l|}{ CHIA-PIFO } \\
\hline & Tissue & -11.88 & 4.85 & 0.0184 \\
\hline \multicolumn{5}{|l|}{ ELAVL-TIMM44 } \\
\hline & Tissue & -13.58 & 4.44 & 0.004 \\
\hline \multicolumn{5}{|l|}{ FAM162B-ZUFSP } \\
\hline & Tissue & -18.69 & 1.87 & $1.45 \times 10^{-10}$ \\
\hline & Sex & -4.982 & 2.00 & 0.019 \\
\hline & Stage & 6.945 & 1.96 & 0.001 \\
\hline \multicolumn{5}{|l|}{ IFNAR2-IL10RB } \\
\hline & Tissue & -10.25 & 4.33 & 0.024 \\
\hline \multicolumn{5}{|l|}{ INMT-FAM188B } \\
\hline & Tissue & -20.33 & 3.65 & $2.43 \times 10^{-6}$ \\
\hline \multicolumn{5}{|l|}{ KIAA1841-C2orf74 } \\
\hline & Tissue & -16.50 & 3.76 & $1.22 \times 10^{-4}$ \\
\hline \multicolumn{5}{|l|}{ NFATC3-PLA2G15 } \\
\hline & Tissue & -15.23 & 4.40 & 0.001 \\
\hline & Sex & -11.23 & 5.28 & 0.040 \\
\hline \multicolumn{5}{|l|}{ SHANK3-ACR } \\
\hline & Tissue & -25.61 & 3.95 & $4.27 \times 10^{-8}$ \\
\hline \multicolumn{5}{|l|}{ SIRPB 1-SIRPD } \\
\hline & Tissue & -18.94 & 3.76 & $2.29 \times 10^{-5}$ \\
\hline
\end{tabular}

${ }^{1}$ Quantitative levels were rank-transformed to improve the normality distribution of values.

${ }^{2}$ Tumor tissue as compared to non-involved tissue; males as compared to females; ever smoker as compared to never smoker; stage $>I$ as compared to stage $=I$.

${ }^{3} \mathrm{SE}$, standard error.

${ }^{4} P$ values are reported only if $P<0.05$; CTSC-RAB38 showed $P>0.05$ for all variables.

Further studies are needed to clarify whether the read-through transcripts identified here are translated into proteins or exert a regulatory function in normal lung tissue.

Until now, read-through transcripts were typically found to be expressed at higher levels in tumor tissue of different cancer types than in the normal tissue counterpart $[13,14,17,25]$, leading to the suggestion that read-through events were involved in carcinogenesis and tumor cell growth. Here, we show that chimeric transcripts, produced by the transcriptional read-through of two adjacent genes, are frequently present in normal lung tissue; a similar phenomenon has recently been observed in human prostate cells [18]. Interestingly, nine of the read-through transcripts identified here were significantly down-regulated in lung adenocarcinoma tissue.

Multivariate statistical modeling did not find any influence of smoking habit on the expression levels of the read-through transcripts. Therefore, it is unlikely that these read-through transcripts originate from some alteration in transcription caused in lung tissue by tobacco smoking. Also sex and tumor stage did not influence read-through transcript levels, except for FAM162B-ZUFSP which had a significant association with both covariates and for NFATC3-PLA2G15 whose levels were associated with sex. These results suggest that sex and stage may play only a minor role, if any, in the mechanisms behind our finding of a down-regulation of these read-through transcript in lung adenocarcinoma tissue. Therefore, after excluding smoking habit, sex and stage as confounders, we speculate that the down-regulation of these read-through transcripts in tumor tissue may be involved in the pathogenetic mechanism of lung tumorigenesis.

For five of the nine significantly down-regulated read-through transcripts (CHIA-PIFO, IFNAR2-IL10RB, NFATC3-PLA2G15, SIRPB 1-SIRPD, and SHANK3-ACR), the reading frames of the two parent genes were maintained, suggesting that a chimeric protein could be produced. It will be interesting to investigate the existence of such proteins in normal lung tissue and in tumor tissue, and to determine if they have any functional role in lung adenocarcinoma pathogenesis. For the other read-through transcripts, where the reading frame was not conserved and a premature stop codon was introduced, it will be interesting to investigate a possible regulatory 
role in the expression of the protein encoded by the first parent gene. Indeed, it has been suggested that these kinds of read-through events suppress the expression of the upstream gene through the nonsense-mediated decay mechanism [10, 29].

In summary, this report documents the existence of read-through transcripts in normal lung parenchyma that are down-regulated in tumor tissues of adenocarcinoma patients. The possible functions of these read-through transcripts and their role in lung tumorigenesis remain to be elucidated.

\section{MATERIALS AND METHODS}

\section{Tissue and RNA samples}

This study used tissue samples held in a biobank at Fondazione IRCCS Istituto Nazionale dei Tumori, Milan, Italy. The samples consisted of lung adenocarcinoma and matched non-involved (apparently normal) lung parenchyma excised from patients who underwent lobectomy for lung adenocarcinoma in the authors' institutes. Methods for the collection of samples and associated clinical data have already been reported [19]. Collection of tissue samples and clinicopathological information from patients had been undertaken with ethical review board approval and informed consent in accordance with the tenets of the Declaration of Helsinki. Specifically, the study protocol was approved by the Committees for Ethics of the institutes involved in recruitment (Fondazione IRCCS Istituto Nazionale dei Tumori, San Giuseppe Hospital, IRCCS Fondazione Cà Granda Ospedale Maggiore Policlinico).

For this study, we used RNA from 103 samples of non-involved lung tissue (64 for RNA-Seq and 45, including 6 of those used for RNA-Seq, for quantitative PCR) and 45 samples of lung adenocarcinoma. The RNA had been extracted for previous studies [19, 30-32]. The clinical characteristics of the corresponding 103 patients are reported in Table 1.

\section{RNA-Seq and identification of read-through transcripts}

RNA-Seq was carried out for 64 samples of non-involved lung tissue as previously described [19]. Qseq files, containing the raw sequencing data, were de-indexed and converted to the Sanger FastQ file format. FastQ sequences were aligned to the human genome assembly (GRCh37/hg19 version) using TopHat v.1.2.0 software [33]. The resulting Sequence Alignment/Map (SAM) files were quality-tested using SAM-Profiler software [34], and only those with high mean read quality ( $>30$ Phred) were analyzed with FusionAnalyser software [35]. Candidate chimeric events were considered all those detected by $\geq 10$ independent reads with a mean read quality $\geq 25$ and with at least 1 read mapping across the predicted exonic breakpoint. These events were further filtered according to their reciprocal mapping and strandness: only adjacent genes mapping to the same gDNA strand were considered as correct read-through predictions and subsequently tested by Sanger sequencing.

\section{Amplification and Sanger sequencing}

cDNA was synthesized, from the 64 RNA samples ( $1 \mu \mathrm{g}$ each) analyzed by RNA-Seq, using the SuperScript VILO cDNA Synthesis Kit (Life Technologies, Foster City, CA, USA). To verify the existence of the identified read-through transcripts in non-involved lung tissue, we amplified the junction regions, using cDNA from at least one sample in which the transcripts were first identified by RNA-Seq. PCR reactions were performed using $40 \mathrm{ng}$ cDNA, 0.6 U AmpliTaq Gold DNA Polymerase (Life Technologies), $0.2 \mu \mathrm{M}$ primers spanning the junction between transcripts (sequences available upon request), $1.5 \mathrm{mM} \mathrm{MgCl}_{2}, 0.1 \mathrm{mM}$ dNTPs, in a final volume of $25 \mu \mathrm{l}$. Amplified fragments were visualized on $4 \%$ agarose gels together with $\Phi \times 174$ DNA-Hae III digest used as DNA molecular weight marker. Amplicons were Sanger-sequenced at Eurofins MWG Operon (Ebersberg, Germany), to verify the sequence at the transcript fusion site.

\section{Quantitative PCR}

RNA (1 $\mu \mathrm{g})$ from 45 pairs of non-involved lung tissue and corresponding lung adenocarcinoma tissue was used to synthesize cDNA by reverse transcription using the Transcriptor First Strand cDNA Synthesis Kit (Roche, Basel, Switzerland). To measure expression of the read-through transcripts in tissue pairs, we did quantitative PCR (qPCR) assays with $12.5 \mathrm{ng}$ cDNA template diluted in RNase-free water, $5 \mu \mathrm{l} 2 \times$ Fast SYBR Green Master Mix (Life Technologies), and $0.3 \mu \mathrm{M}$ primers (sequences available upon request; these primers differed from those used to validate read-through fusion points, since qPCR amplicons must be relatively short, i.e. 70-100 bp, whereas PCR amplicons for Sanger sequencing need to be longer) in a final volume of $10 \mu \mathrm{l}$. The human hypoxanthine phosphoribosyltransferase 1 (HPRT1) gene was used to normalize expression data. Reactions were run in duplicate using the QuantStudio 12K Flex or 7900 HT Fast real-time PCR system (Life Technologies). We considered as unreliable, and thus excluded, qPCR data of those pairs in which either the non-involved or tumor sample, or both, had a cycle threshold $(\mathrm{Ct})$ standard deviation $>0.5$. For this reason, a different number of analyzed pairs is reported for each read-through transcript in Figure 1. RQ values for each read-through transcript were calculated, with ExpressionSuite Software v1.0.4 (Life Technologies), with respect to the RQ of the same transcript measured in 
a calibrator sample, composed of a pool of cDNAs from normal lung tissue samples previously found by RNA-Seq to be positive for the measured read-through transcripts.

\section{Statistical analyses}

Differences in transcript expression levels between normal and tumor tissues were tested for significance using the non-parametric Wilcoxon's signed-rank test for paired samples. The effects of clinical characteristics on transcript levels were tested for significance in a multivariate linear model on rank-transformed data [36]. Both statistical tests were executed using the $\mathrm{R}$ graphical user interface "R Commander" [37].

\section{ACKNOWLEDGMENTS}

We thank Dr. Valerie Matarese for scientific editing. AD was recipient of an AIRC/FIRC fellowship, Rif.16353.

\section{CONFLICTS OF INTEREST}

The authors declare no conflicts of interest.

\section{GRANT SUPPORT}

AD is recipient of the AIRC/FIRC fellowship no. 16353. This work was supported in part by grants from the Italian Association for Cancer Research (AIRC, grants no. 10323 and 12162). The funders had no role in the design and conduct of the study, in the collection, analysis, and interpretation of the data, and in the preparation, review, or approval of the manuscript.

\section{REFERENCES}

1. Seo JS, Ju YS, Lee WC, Shin JY, Lee JK, Bleazard T, Lee J, Jung YJ, Kim JO, Shin JY, Yu SB, Kim J, Lee ER, et al. The transcriptional landscape and mutational profile of lung adenocarcinoma. Genome Res. 2012; 22:2109-2119.

2. Forde PM, Ettinger DS. Targeted therapy for non-small-cell lung cancer: Past, present and future. Expert Rev Anticancer Ther. 2013; 13:745-758.

3. Sholl LM, Aisner DL, Varella-Garcia M, Berry LD, DiasSantagata D, Wistuba II, Chen H, Fujimoto J, Kugler K, Franklin WA, Iafrate AJ, Ladanyi M, Kris MG, et al. Multiinstitutional oncogenic driver mutation analysis in lung adenocarcinoma: The lung cancer mutation consortium experience. J Thorac Oncol. 2015; 10:768-777.

4. Lee SE, Lee B, Hong M, Song JY, Jung K, Lira ME, Mao M, Han J, Kim J, Choi YL. Comprehensive analysis of RET and ROS1 rearrangement in lung adenocarcinoma. Mod. Pathol. 2015; 28:468-479.
5. Fan L, Feng Y, Wan H, Shi G, Niu W. Clinicopathological and demographical characteristics of non-small cell lung cancer patients with ALK rearrangements: A systematic review and meta-analysis. PLoS One. 2014; 9:e100866.

6. Soda M, Choi YL, Enomoto M, Takada S, Yamashita Y, Ishikawa S, Fujiwara S, Watanabe $\mathrm{H}$, Kurashina $\mathrm{K}$, Hatanaka H, Bando M, Ohno S, Ishikawa $\mathrm{Y}$, et al. Identification of the transforming EML4-ALK fusion gene in non-small-cell lung cancer. Nature. 2007; 448:561-566.

7. Takeuchi K, Soda M, Togashi Y, Suzuki R, Sakata S, Hatano S, Asaka R, Hamanaka W, Ninomiya H, Uehara H, Lim Choi Y, Satoh Y, Okumura S, et al. RET, ROS1 and ALK fusions in lung cancer. Nat Med. 2012; 18:378-381.

8. Bergethon K, Shaw AT, Ou SH, Katayama R, Lovly CM, McDonald NT, Massion PP, Siwak-Tapp C, Gonzalez A, Fang R, Mark EJ, Batten JM, Chen H, et al. ROS1 rearrangements define a unique molecular class of lung cancers. J Clin Oncol. 2012; 30:863-870.

9. Kohno T, Ichikawa H, Totoki Y, Yasuda K, Hiramoto M, Nammo T, Sakamoto H, Tsuta K, Furuta K, Shimada Y, Iwakawa R, Ogiwara H, Oike T, et al. KIF5B-RET fusions in lung adenocarcinoma. Nat Med. 2012; 18:375-377.

10. Akiva P, Toporik A, Edelheit S, Peretz Y, Diber A, Shemesh R, Novik A, Sorek R. Transcription-mediated gene fusion in the human genome. Genome Res. 2006; 16:30-36.

11. Wang Q, Xia J, Jia P, Pao W, Zhao Z. Application of next generation sequencing to human gene fusion detection: Computational tools, features and perspectives. Brief Bioinform. 2013; 14:506-519.

12. Nacu S, Yuan W, Kan Z, Bhatt D, Rivers CS, Stinson J, Peters BA, Modrusan Z, Jung K, Seshagiri S, Wu TD. Deep RNA sequencing analysis of readthrough gene fusions in human prostate adenocarcinoma and reference samples. BMC Med. Genomics. 2011; 4:11-8794-4-11.

13. Kim HP, Cho GA, Han SW, Shin JY, Jeong EG, Song SH, Lee WC, Lee KH, Bang D, Seo JS, Kim JI, Kim TY. Novel fusion transcripts in human gastric cancer revealed by transcriptome analysis. Oncogene. 2014; 33:5434-5441.

14. Varley KE, Gertz J, Roberts BS, Davis NS, Bowling KM, Kirby MK, Nesmith AS, Oliver PG, Grizzle WE, Forero A, Buchsbaum DJ, LoBuglio AF, Myers RM. Recurrent readthrough fusion transcripts in breast cancer. Breast Cancer Res Treat. 2014; 146:287-297.

15. Zhang H, Lin W, Kannan K, Luo L, Li J, Chao PW, Wang Y, Chen YP, Gu J, Yen L. Aberrant chimeric RNA GOLM1MAK10 encoding a secreted fusion protein as a molecular signature for human esophageal squamous cell carcinoma. Oncotarget. 2013; 4:2135-2143. doi: 10.18632/oncotarget.1465.

16. Zhang Y, Gong M, Yuan H, Park HG, Frierson HF, Li H. Chimeric transcript generated by cis-splicing of adjacent genes regulates prostate cancer cell proliferation. Cancer Discov. 2012; 2:598-607. 
17. Pflueger D, Mittmann C, Dehler S, Rubin MA, Moch H, Schraml P. Functional characterization of BC039389GATM and KLK4-KRSP1 chimeric read-through transcripts which are up-regulated in renal cell cancer. BMC Genomics. 2015; 16:247.

18. Qin F, Song Z, Babiceanu M, Song Y, Facemire L, Singh R, Adli M, Li H. Discovery of CTCF-sensitive cis-spliced fusion RNAs between adjacent genes in human prostate cells. PLoS Genet. 2015; 11:e1005001.

19. Galvan A, Frullanti E, Anderlini M, Manenti G, Noci S, Dugo M, Ambrogi F, De Cecco L, Spinelli R, Piazza R, Pirola A, Gambacorti-Passerini C, Incarbone $\mathrm{M}$, et al. Gene expression signature of non-involved lung tissue associated with survival in lung adenocarcinoma patients. Carcinogenesis. 2013; 34:2767-2773.

20. Frenkel-Morgenstern M, Lacroix V, Ezkurdia I, Levin Y, Gabashvili A, Prilusky J, Del Pozo A, Tress M, Johnson R, Guigo R, Valencia A. Chimeras taking shape: Potential functions of proteins encoded by chimeric RNA transcripts. Genome Res. 2012; 22:1231-1242.

21. Parra G, Reymond A, Dabbouseh N, Dermitzakis ET, Castelo R, Thomson TM, Antonarakis SE, Guigo R. Tandem chimerism as a means to increase protein complexity in the human genome. Genome Res. 2006; 16:37-44.

22. Prakash T, Sharma VK, Adati N, Ozawa R, Kumar N, Nishida Y, Fujikake T, Takeda T, Taylor TD. Expression of conjoined genes: Another mechanism for gene regulation in eukaryotes. PLoS One. 2010; 5:e13284.

23. Kim RN, Kim A, Choi SH, Kim DS, Nam SH, Kim DW, Kim DW, Kang A, Kim MY, Park KH, Yoon BH, Lee KS, Park HS. Novel mechanism of conjoined gene formation in the human genome. Funct. Integr. Genomics. 2012; $12: 45-61$.

24. Frenkel-Morgenstern M, Valencia A. Novel domain combinations in proteins encoded by chimeric transcripts. Bioinformatics. 2012; 28:i67-74.

25. Yun SM, Yoon K, Lee S, Kim E, Kong SH, Choe J, Kang JM, Han TS, Kim P, Choi Y, Jho S, Yoo H, Bhak J, et al. PPP1R1B-STARD3 chimeric fusion transcript in human gastric cancer promotes tumorigenesis through activation of PI3K/AKT signaling. Oncogene. 2014; 33:5341-5347.

26. Djebali S, Lagarde J, Kapranov P, Lacroix V, Borel C, Mudge JM, Howald C, Foissac S, Ucla C, Chrast J, Ribeca P, Martin D, Murray RR, et al. Evidence for transcript networks composed of chimeric RNAs in human cells. PLoS One. 2012; 7:e28213.
27. Pradet-Balade B, Medema JP, Lopez-Fraga M, Lozano JC, Kolfschoten GM, Picard A, Martinez-A C, Garcia-Sanz JA, Hahne M. An endogenous hybrid mRNA encodes TWEPRIL, a functional cell surface TWEAK-APRIL fusion protein. EMBO J. 2002; 21:5711-5720.

28. Thomson TM, Lozano JJ, Loukili N, Carrio R, Serras F, Cormand B, Valeri M, Diaz VM, Abril J, Burset M, Merino J, Macaya A, Corominas M, et al. Fusion of the human gene for the polyubiquitination coeffector UEV1 with kua, a newly identified gene. Genome Res. 2000; 10:1743-1756.

29. Poulin F, Brueschke A, Sonenberg N. Gene fusion and overlapping reading frames in the mammalian genes for 4E-BP3 and MASK. J Biol Chem. 2003; 278:52290-52297.

30. Frullanti E, Colombo F, Falvella FS, Galvan A, Noci S, De Cecco L, Incarbone M, Alloisio M, Santambrogio L, Nosotti M, Tosi D, Pastorino U, Dragani TA. Lung adenocarcinoma clinical stage is associated with gene expression pattern in non-involved lung tissue. Int J Cancer. 2012.

31. Galvan A, Noci S, Taverna F, Lombardo C, Franceschi S, Pastorino U, Dragani TA. Testing of human papillomavirus in lung cancer and non-tumor lung tissue. BMC Cancer. 2012; 12:512-2407-12-512.

32. Galvan A, Colombo F, Frullanti E, Dassano A, Noci S, Wang Y, Eisen T, Matakidou A, Tomasello L, Vezzalini M, Sorio C, Dugo M, Ambrogi F, et al. Germline polymorphisms and survival of lung adenocarcinoma patients: A genomewide study in two european patient series. Int J Cancer. 2015; 136:E262-71.

33. Trapnell C, Pachter L, Salzberg SL. TopHat: Discovering splice junctions with RNA-seq. Bioinformatics. 2009; 25:1105-1111.

34. Flora F, Pirola A, Spinelli R, Redaelli S, Valletta S, Gambacorti-Passerini C, Piazza R. SAM-profiler: A graphical tool for qualitative profiling of next generation sequencing alignment data. J Comput Sci Syst. Biol. 2013; 6:188-193.

35. Piazza R, Pirola A, Spinelli R, Valletta S, Redaelli S, Magistroni V, Gambacorti-Passerini C. FusionAnalyser: A new graphical, event-driven tool for fusion rearrangements discovery. Nucleic Acids Res. 2012; 40:e123.

36. Conover WJ, Iman RL. Rank transformations as a bridge between parametric and nonparametric statistics. Am Statistician. 1981; 35:124-129.

37. Fox J. The R commander: A basic-statistics graphical user interface to R J Stat. Software. 2005; 19:1-42. 